\title{
Diagnóstico tecnológico y socioeconómico de los cultivos de maracuyá y guayaba en el Ariari, Meta.
}

\section{Technological and socioeconomic diagnosis of passion fruit and guava crops in Ariari, Meta.}

\author{
Ana Cecilia Romero-Ramirez , Maira Alejandra Salazar-Cerón² y Javier Orlando Orduz-Rodriguez ${ }^{3}$
}

Recibido para publicación: Septiembre 7 de 2018 - Aceptado para publicación: Noviembre 23 de 2018

\begin{abstract}
RESUMEN
La fruticultura es la principal actividad agrícola de la región del Ariari en el departamento del Meta. Los cultivos de guayaba (Psidium guajava L.) y maracuyá (Passiflora edulis Sims) se establecieron en la década de 1990 y actualmente se siembran cerca de 2000 hectáreas de cada cultivo. Con el fin de conocer los principales problemas fitosanitarios y las prácticas de manejo utilizadas por los productores se realizó un estudio con una encuesta a 40 productores de guayaba y a 43 de maracuyá en tres municipios del Meta (Granada, Lejanías y Villavicencio). La información recolectada se socializó con las comunidades y los resultados se validaron en talleres grupales. Los productores no tienen apoyo de servicios de asistencia técnica ni de extensión agrícola. No se tienen criterios técnicos para los niveles críticos para el control de plagas y enfermedades, ni de los productos ni dosis para su control; además de utilizar productos no recomendados para estos cultivos ni para los problemas fitosanitarios. Estos métodos de manejo elevan los costos de producción y las frutas pueden presentar problemas con los residuos químicos. Se requiere la implementación de capacitación de los productores y la evaluación de control biológico de plagas y de prácticas de agricultura limpia y el acompañamiento de programas de extensión especializado en fruticultura
\end{abstract}

Palabras clave: Mejoramiento, híbridos, órganos reproductivos de plantas.

\begin{abstract}
Fruit growing is the main agricultural activity of the Ariari region in the department of Meta. The cultivations of guava (Psidium guajava L.) and passion fruit (Passiflora edulis Sims) were established in the 1990s and currently about 2000 hectares of each crop are sown. In order to know the main phytosanitary problems and their management practices, a study was conducted with a survey of 40 guava producers and 43 maracuya producers in the Meta municipalities (Granada, Lejanías and Villavicencio). The information collected was socialized with the communities and the results were validated in-group workshops. The producers do not have support for technical assistance or agricultural extension services. We do not have the same technical means for the control of pests and diseases, neither the products nor the dose for their control; In addition to using products not recommended for these products or for phytosanitary problems. These management methods are higher than production costs and fruits. The implementation of the training of the producers and the evaluation of the biological control of the plagues and the practices of clean agriculture and the accompaniment of the programs of extension specialized in fruitculture is required
\end{abstract}

Key words: Carnation, breeding, hybrids, plant reproductive organs.

\footnotetext{
${ }^{1}$ Ph.D. Universidade Federal de Viçosa acero1167@yahoo.com

${ }^{2}$ Bióloga Universidad de los Llanos

3* Ph.D. Agrosavia. Centro de Investigación La Libertad. Villavicencio, Meta. jorduz@agrosavia.co
} 


\section{INTRODUCCIÓN}

Históricamente, la agricultura ha jugado un papel esencial en el desarrollo económico de las naciones. Tanto en países desarrollados como en economías emergentes, este ha sido el motor de crecimiento de los demás sectores (Pingali 2007; Perfetti et al., 2013). Según el DANE-CNA (2016), en el año 2014 los cultivos frutales representan el $14,6 \%$ de las hectáreas cultivadas del país, ocupando el cuarto renglón en importancia a nivel nacional.

De estos, el cultivo de guayabo (Psidium guajava), es considerado de importancia comercial en más de 60 países tropicales y subtropicales al ser usado como fuente de alimento y de medicina popular (Salazar et al. 2006). En Colombia el área cosechada del cultivo fue de 6.907 hectáreas para el año 2016, con una producción de 70.054 toneladas, y el departamento del Meta en el mismo año reporta una producción de 40,00 toneladas en un área cercana a las 2000 hectáreas (Agronet, 2017). En cuanto al cultivo de maracuyá (Passiflora edulis), su fruto es utilizado para su consumo como bebida refrescante y en algunos países se utilizan las flores como infusión con fines medicinales (Zibadi \& Watson, 2004). En cuanto a la producción de este cultivo, en el año 2016 a nivel nacional se produjeron $119.388,87$ t. correspondientes a $7.192,30$ ha de cultivo de las cuales en el Meta se produce una quinta parte de la producción, oscilando el área entre 1500 a 2000 hectáreas.

Cerca del $90 \%$ de los cultivos de guayaba y maracuyá sembrados en el departamento del Meta se encuentran ubicados en los municipios de Granada, Lejanías, Fuente de Oro, Puerto Lleras, El Dorado Y El Castillo. Su Producción está orientada a abastecer el mercado de Bogotá y su comercialización se realiza principalmente con los intermediarios de Corabastos quienes recogen la fruta directamente en las fincas productivas. Estos cultivos son una importante fuente de empleo e ingresos para las comunidades productoras. Sin embargo, la ausencia de oferta tecnológica, conocimiento y capacitación impiden aprovechar el potencial de rendimiento de los cultivos y tener prácticas de manejo fitosanitario con criterios técnicos, ambientales y económicos adecuados. Para ahondar más en el conocimiento de esta problemática, se realiza la siguiente investigación cuyo propósito es; conocer la condición socioeconómica de los productores y el manejo tecnológico de los cultivos de maracuyá y guayaba de la región del Ariari, Meta.

\section{MATERIALES Y MÉTODOS}

Se realizó un diagnóstico participativo de los aspectos socio económicos, fitosanitarios y tecnológicos de los cultivos de guayaba y maracuyá en 40 fincas productoras de guayaba y 43 de maracuyá, ubicadas en los municipios de Granada, Lejanías y Villavicencio, departamento del Meta. Para este propósito se recopiló información en campo a través de entrevistas individuales a una muestra representativa de productores de ambos cultivos. La información sobre la población de productores, se obtuvo a través de consultas con la Secretaría de Agricultura del Meta, cadena frutícola del Meta y representantes de asociaciones de productores. Con base en esta población, se seleccionó una muestra aleatoria estratificada según el tamaño del cultivo y que representara el 30\% de la población, mediante la fórmula de muestreo probabilístico:

$$
P=n / N
$$

$\mathrm{P}$ : es muestreo probabilístico aleatorio simple, $\mathrm{n}$ : muestra y $\mathrm{N}$ : universo.

Las encuestas se aplicaron a los productores en cada finca y se georeferenciaron los predios. Los datos se analizaron mediante estadística descriptiva, utilizando 69 variables agrupadas en aspectos socioeconómicos, tecnológicos y fitosanitarios. Dentro de las 
variables utilizadas se puede mencionar nivel de escolaridad, ingresos familiares, área de siembra, época de establecimiento de los cultivos, distancias de siembra, prácticas de manejo tecnológico del cultivo, labores de cosecha y poscosecha, manejo de agroinsumos y control de plagas y enfermedades, entre otras. Esta información obtenida en campo y los resultados de identificación de plagas y enfermedades realizados en laboratorios de centros de investigación se validaron en talleres de diagnóstico participativo con los productores mediante discusión en plenaria. Así fueron priorizados los principales problemas fitosanitarios para estos cultivos en la región, las limitantes de estos y posibles soluciones planteadas por los productores.

\section{RESULTADOS Y DISCUSIÓN}

La ubicación por municipios de las fincas evaluadas para el cultivo de guayaba el $72,5 \%$ de ellas se encuentran en el municipio de Lejanías y en menor proporción $(27,5 \%)$ en el municipio de Granada, ambos municipios ubicados en el piedemonte del Meta. Las fincas encuestadas con cultivos de maracuyá están ubicadas en el municipio de Granada con un porcentaje del $60,5 \%$, en Lejanías estaban el $32,6 \%$ de las fincas encuestadas; ambas localidades de la región del Ariari y en Villavicencio se encuestaron el $7 \%$ del total de las fincas.

Condiciones sociodemográficas de los cultivadores. Para ambos cultivos el $66,6 \%$ de los productores son hombres y el 33,4\% mujeres muy cercano a los promedios nacionales reportados por el DANE-CNA (2016). En cuanto al nivel de escolaridad, la educación primaria es la que predomina en la zona, fenómeno que a nivel nacional representa el 57,4\% y en el Meta corresponde al 58,5\% (DANECNA, 2016). De igual forma, la mayoría de personas encuestadas superan los 45 años de edad, lo cual se ve reflejado en los resultados del DANE donde demuestra que el $96,3 \%$ de las personas productoras a nivel nacional tienen 45 años o más (DANE-CNA, 2016). El tamaño general de las fincas productivas fue de 3,0 ha y los agricultores son los propietarios de estas. El tamaño promedio de los lotes cultivados en guayaba es de 2,0 ha y el de los lotes de maracuyá es menor a 1,0 ha; además, se encontraron algunos cultivos asociados con cítricos o plátano principalmente. Los productores cuentan, por lo menos, con cuatro años de experiencia en el manejo de estos cultivos. Estos datos son comparables con los datos del DANE-CNA (2016) donde se demuestra que el $69,5 \%$ de las Unidades Productivas Agrícolas - UPA son menores de 5 ha y ocupan el $5,2 \%$ del territorio dedicado a producción.

A pesar del tamaño reducido, los productores consideran que estos cultivos son rentables. Por último, la mayoría de los productores no se encuentran adscritos a asociaciones de productores. De los asociados el mayor número son los de guayaba, los cuales pertenecen a Asodafari, Asoguayaba, Aspromercary Asofrutal; mientras que los de maracuyá, pertenecen a Frutorinoquía, Asomari, Garzón Apache, Asohofrucol y Sebastopol. A nivel nacional el $73,7 \%$ de los productores no está asociado, $y$ en el Meta el 81,4\% (DANE-CNA, 2016).

Manejo tecnológico de los cultivos de guayaba y maracuyá. Los productores de guayaba no realizan adecuaciones del lote antes de la siembra; mientras que los de maracuyá hacen actividades de drenaje, encamado, arado y rastrillo. En ambos casos, el material de siembra y vegetal utilizado proviene principalmente de viveros no certificados de la zona o plántulas obtenidas en las fincas de los productores. Este último es un factor importante en la aparición de plagas y enfermedades durante la etapa productiva, ya que la selección de semillas o de material vegetal que no certifique su procedencia, puede encontrarse infectado o infestado con algún tipo de ente patógeno y provocar su dispersión 
en todo el cultivo (ICA, 2011; ICA, 2012). El $74 \%$ de los productores expresa no haber recibido capacitación en el manejo tecnológico de los cultivos y manifiestan que las necesidades más urgentes de capacitación son: en manejo de plagas y enfermedades, así como preparación y uso de abonos orgánicos.

En cuanto al almacenamiento de los agroquímicos y la disposición final de sus residuos, no se siguen las normas de seguridad requeridas. Los productos no se almacenan en cuartos separados de la vivienda, otros los dejan en los lotes cubriéndolos con lonas o guardándolos en bolsas de plástico. Los productores no acostumbran a usar trajes adecuados para la aplicación de agroquímicos, solo usan máscara y botas, pero estos elementos no se revisan o cambian frecuentemente. Estos son factores que incumplen la normatividad en cuanto a las Buenas Prácticas Agrícolas BPA establecidas (Betancurt et al., 2014; Izquierdo \& Rodríguez, 2016), fenómeno que puede ser incluso perjudicial para la salud de los agricultores, ya que según el Banco Mundial (2008) cada año mueren 355.000 personas por intoxicación con plaguicidas, y a su vez afecta la sostenibilidad ambiental por contaminación y afectación a la diversidad de fauna y flora (FAO, 2002; Banco mundial, 2008).

Manejo tecnológico del cultivo de Guayaba En este cultivo la variedad más sembrada es la Guayaba pera de plántulas injertadas, donde la densidad de plantación más utilizada está entre 250 a 300 plantas/ha, lo que corresponde al $46 \%$. Por otra parte, las distancias de plantación varían desde 3 a $8 \mathrm{~m}$ entre surcos y de 4 a $8 \mathrm{~m}$ entre plantas siendo las más usadas de $5 \times 5 \mathrm{~m}$, correspondiente al $36 \%$ de las producciones evaluadas.

El Instituto Colombiano Agropecuario ICA (2012) avala las distancias de $5 \times 5 \mathrm{~m}$, de 4 x $4 \mathrm{~m}$ y de 3,5 x 3,5m como una de las más usadas. De igual forma, según el Centro
Nacional de Tecnología Agropecuaria y Forestal "Enrique Álvarez Córdova" CENTA (2010a), en El Salvador el distanciamiento de siembra recomendado para este cultivo es de 3,75 m entre calle y de 3,0 m entre plantas, con una densidad promedio de siembra de 622 plantas por hectárea. Al comparar los datos obtenidos con las recomendaciones de estos entes avaladores, se observa que la densidad de siembra utilizada por los productores es baja, y aunque aumentarla puede generar costos extra por hectárea, puede favorecer los niveles de productividad, esto siempre y cuando se realice un manejo agronómico adecuado y de sistemas de monitorio y control de plagas y enfermedades eficientes (CENTA, 2010a; ICA, 2012).

Dentro de las labores culturales realizadas en el cultivo de guayaba se encuentran las podas, las deschuponadas y el control de malezas. Las podas varían desde cada 15 días hasta dos por año. La fertilización más utilizada es la química con el uso de Agrimins, Triple 15 y Nutrimins. El riego es usado por el $75 \%$ de los productores, mediante mecanismo de riego por goteo, con agua procedente de pozos o aljibes. Para este cultivo, se sugiere que se debe realizar una adecuación previa del terreno y la respectiva fertilización, la cual depende de los resultados obtenidos en el análisis de suelo (Gómez y Rebolledo, 2011; ICA, 2012). De igual forma, se deben realizar podas de formación para el control fitosanitario (ICA y Corpoica, 2006; ICA, 2012), podas de raleo y podas de fructificación (ICA, 2012).

Manejo tecnológico del cultivo de Maracuyá Para el cultivo de maracuyá, el rango de la densidad de siembra más utilizado en las fincas productivas fue de 1.000 a 1.250 plantas/ha, con una representación del 35,6\%. En cuanto a las distancias de siembra varían desde 1 a $4 \mathrm{~m}$ entre surcos y de 2 a $5 \mathrm{~m}$ entre plantas siendo las más utilizadas $3 \times 3 \mathrm{~m}$ correspondientes al $23 \%$ y las de $4 \times 4 \mathrm{~m}$ con el $20 \%$. Estos valores obtenidos son equiparables con las 
recomendaciones del ICA (2011) quien sugiere siembras de $4 \times 4 \mathrm{~m}$ con el establecimiento de 625 plantas. Entidades internacionales como el CENTA (2010b) concluye que el uso de distancias cortas aumenta el rendimiento en el primer año; sin embargo, a partir del segundo la eficiencia disminuye debido al exceso de masa foliar la cual provoca sombra y deficiencias fotosintéticas de las plantas. Al elegir densidades de siembra mayores es indispensable el uso de un conjunto de recomendaciones eficientes, con riego localizado, polinización manual, reposición de las deficiencias nutricionales, entre otros (Amaya, 2009).

En cuanto a prácticas culturales, en todas las fincas encuestadas se hace control de malezas, fertilización, podas de crecimiento y formación, con una frecuencia quincenal; deshoje, deschuponadas y control de problemas fitosanitarios. En este cultivo, generalmente los productores no utilizan riego $(64,4 \%$ ) y los que lo hacen tienen sistema por goteo y obtienen el agua de quebradas y pozos. El tutorado más usado es el emparrado, seguido por el de espaldera, con uso de madera proveniente de bosques nativos, lo cual ocasiona altos costos del establecimiento de los cultivos y dificultades legales con las entidades del sector ambiental. Por último, los fertilizantes más usados en maracuyá son Triple 15 y Triple 18. Para este caso, se identifica que los agricultores están realizando adecuadamente las labores culturales de acuerdo a lo determinado por los distintos entes (Cadena Productiva Frutícola, 2006; Amaya, 2009; CENTA, 2010b; ICA, 2011; CIAT, 2012; Betancurt et al. 2014).

Condiciones y manejo fitosanitario de los cultivos. El 93\% de los productores no utiliza un sistema de monitoreo de incidencia o severidad de plagas o enfermedades. A su vez, realizan aplicaciones de agroquímicos siguiendo la práctica de tipo calendario con una frecuencia quincenal, la cual se incrementa a semanal o menos en épocas que se consideran de alta incidencia de plagas y enfermedades. La importancia de realizar un monitoreo radica en detectar a tiempo la aparición de plagas y enfermedades, identificarlas y establecer su intensidad, este debe ser un proceso continuo a realizar con una frecuencia semanal o quincenal (Brechelt, 2004; ICA, 2012).

El 96\% de los productores no conocen otras alternativas para el manejo de problemas fitosanitarios en sus cultivos, no cuentan con asistencia técnica y las recomendaciones las reciben de otros productores y de las empresas comercializadoras de agroquímicos. Esta es una tendencia común y marcada en los sistemas de producción agrícola del país, según el DANE-CNA (2016) para el año 2013 el $57,8 \%$ de las UPA realizaban control de plagas y enfermedades con productos químicos. Fenómeno ratificado por la FAO (2002), quien reporta que en los últimos 35 años el uso de plaguicidas ha aumentado considerablemente, logrando tasas de crecimiento de hasta el 5,4 por ciento en algunas regiones. $Y$ por el ICA (2016) quien para el año 2015, contabiliza la producción de agroquímicos en 24,5 millones de kilogramos y 50,9 millones de litros de plaguicidas en el país, demostrando el uso elevado de agroquímicos como controladores. Siendo este último factor, uno de los desencadenes en los procesos de adaptación de las plagas y enfermedades, mediante el uso inadecuado o indiscriminado de agroquímicos (Cadena Productiva Frutícola, 2006).

Condiciones y manejo fitosanitario del cultivo de guayaba Las principales plagas identificadas por los productores de guayaba como las más limitantes fueron: el picudo (Conotrachelus psidii), los ácaros del fruto y del cogollo (Brevipalpus phoenicis, Tegonotus guavae), el chinche patón (Leptoglossus sp.), los nematodos (Meloidogyne sp.) y el gusano entorchador (Platynota sp.). El picudo es la plaga más frecuente en las fincas productivas evaluadas, con una presencia del $67 \%$, 
seguida por los ácaros con un 20\% (Figura 1). En cuanto a las enfermedades el mayor porcentaje correspondió a hongos $(97 \%)$ y en menor porcentaje $(3 \%)$ mencionan las causadas por bacterias. De los hongos identificados, la pestalotia (Pestalotiopsis psidii), roña (Puccini psidii Wint), antracnosis (Colletorichum sp.) y fumagina (Capnodium sp.) (Figura 2). El control de estas plagas y enfermedades se hace principalmente mediante el uso de agroquímicos comerciales. Los ingredientes activos de los plaguicidas que más se utilizan son: Fipronil (63\%), Abamectina $(20 \%)$, Carbofuran $(10 \%)$ y Monocrotophos (10\%); seguido del Clorpirifos, Malathion, Cipermetrina, Profenofos, Lambdacihalotrina, Bromopropilato, Metomyl, Thiametoxan, Methil paration y el Milbemectin, (Figura 2). Para el caso del control de las enfermedades se utilizan agroquímicos principalmente con los siguientes ingredientes activos: Mancozeb (75\%), Azufre $(23 \%)$, Carbendazim $(8 \%)$, Propineb (8\%) y en menor medida Benomil, Captan, y Oxicloruro de cobre (Figura 3).

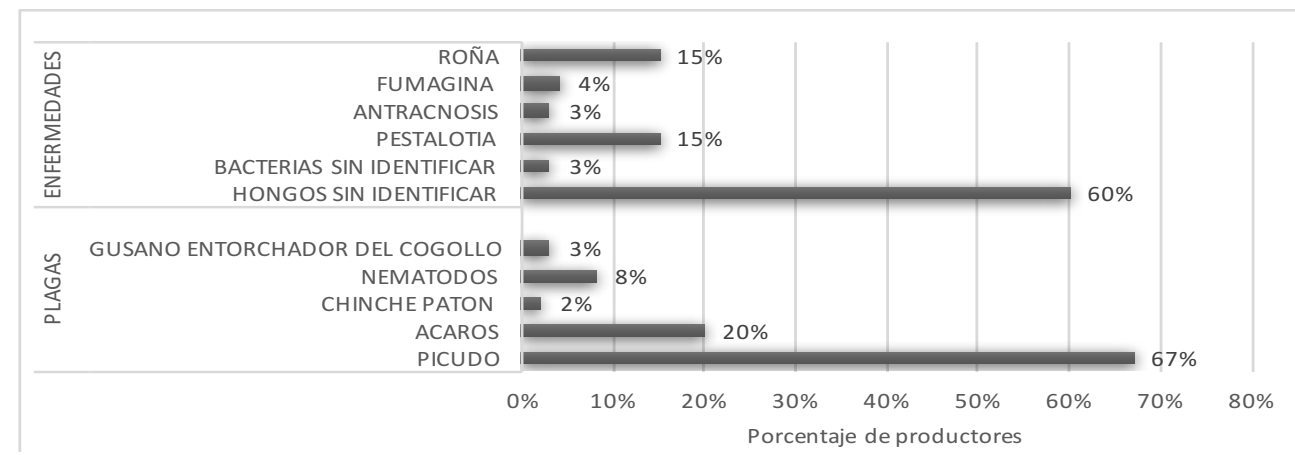

Figura 1. Principales plagas y enfermedades en el cultivo de maracuyá, según lo descrito por los agricultores de la región del Ariari, 2011. Fuente: Los autores..

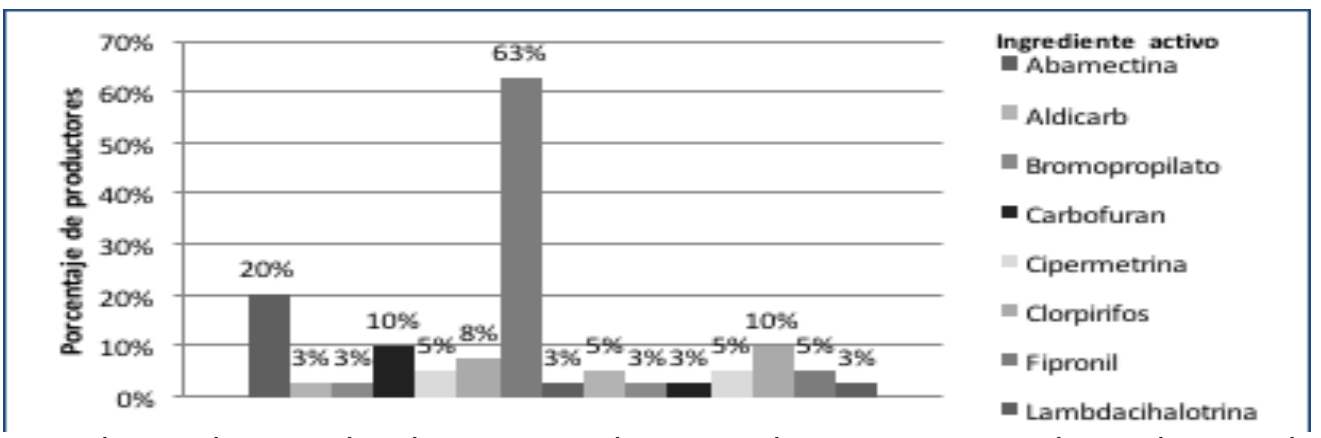

Figura 2. Plaguicidas utilizados por los productores en el cultivo de guayaba en la región del Ariari, departamento del Meta. 2011. Fuente: Los autores..

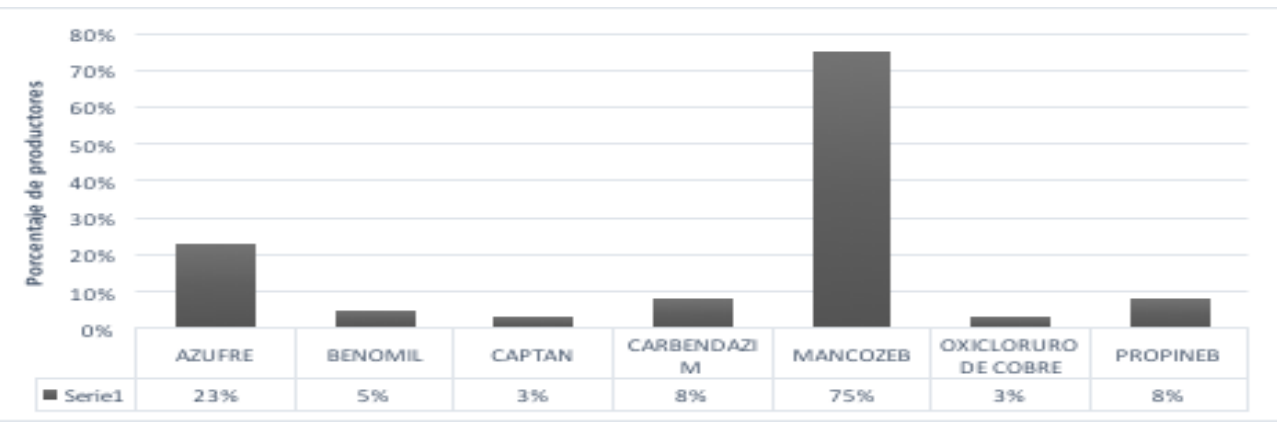

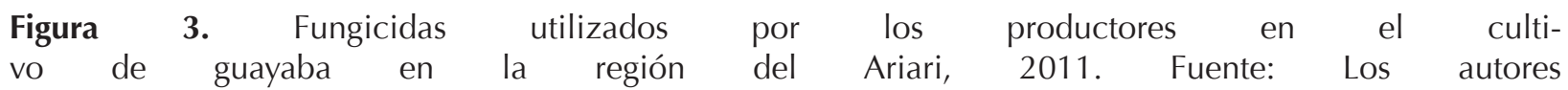


Las afectaciones al cultivo, por la acción de las distintas plagas y enfermedades descritas, demeritan la calidad de las producciones, generan pérdidas y sobrecostos (ICA, 2012). Y aunque los productores demuestran, mediante sus experiencias, que el uso de los agroquímicos controla la mayor parte de estas afectaciones, existen estudios que demuestran la efectividad de los agentes de control biológico, como los descritos por Prakash \& Pandey (2007) y El-argawy (2016) específicamente en plagas y enfermedades de este cultivo. Para el caso del control de nematodos los agricultores encuestados indican la dificultad de generar un control efectivo, no obstante, autores como Zhang et al. (2015); Escudero et al. (2016); Kaur et al. (2016) afirman que existe la posibilidad de controlarlos mediante el uso de microrganismos con capacidad nematófaga.

Condiciones y manejo fitosanitario del cultivo de maracuyá Las principales plagas identificadas por los productores en el cultivo de maracuyá fueron la mosca del ovario (Dasiops sp.) identificada por el $55 \%$ de ellos y el grajo o chinche patón (Leptoglossus sp. y Corythucha gossypii) con el $26 \%$ de representatividad; en menor medida, los agricultores identifican otras plagas tales como: los ácaros (Tetranichus sp.), los áfidos (Aphis gossipii, Myzus persicae), los trips (Thrips tabaci Lindeman, Frankliniella auripes Hood, Frankliniella occidentalis
Perg., Neohydatothrips signifer Priesner) y el gusano defoliador (Agraulis sp.) (Figura 4). Las principales enfermedades identificadas por los productores en el cultivo de maracuyá fueron la antracnosis (Colletotrichum gloesporioides), identificada por el $83 \%$ de los productores, la mancha parda (Alternaria passiflorae), la secadera (Fusarium sp.), la bacteriosis y un mínimo porcentaje la roña (Cladosporium sp.) (Figura 4).

Para el control de las plagas en este cultivo, el $26 \%$ de los productores aplica cebos tóxicos, los cuales se elaboran con melaza, Methil parathion y urea principalmente; en estas preparaciones también se utilizan insecticidas como Tedhion y Clorpirifos. Los otros ingredientes activos de los plaguicidas que utilizan son Cipermetrina $(14 \%)$, Dimetoato $(12 \%)$, Methil parathion $(9 \%)$, y Lambdacihalotrina $(7 \%)$, un número menor de productores utilizan Monocrotofos (Figura 5). Vale la pena resaltar que varios de estos productos son altamente tóxicos y algunos, como el Carbofuran, Monocrotophos y Methil parathion, se encuentran prohibidos para su uso y comercialización desde 1996, ratificado por el Convenio de Rotterdam del año 2004 (de Rotterdam, 2004). Para el control de las enfermedades, los fungicidas más utilizados son: Mancozeb (33\%), Carbendazim (19 $\%)$, Benomil (10\%), Cloruro de Cobre (8\%), Propineb (6\%) y Mefenoxam (5\%) (Figura 6).

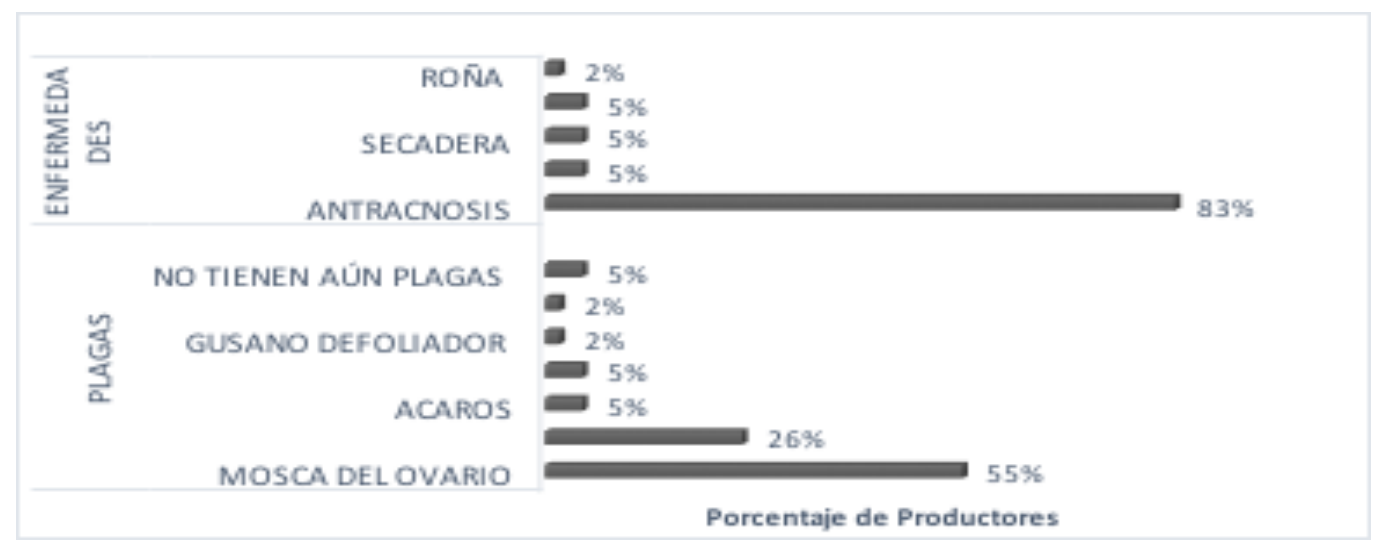

Figura 4 Principales plagas y enfermedades en el cultivo de maracuyá, según lo descrito por los agricultores de la región del Ariari, 2011. Fuente: Los autores. 
Romero-Ramirez et al. - Maracuyá y guayaba en el Ariari

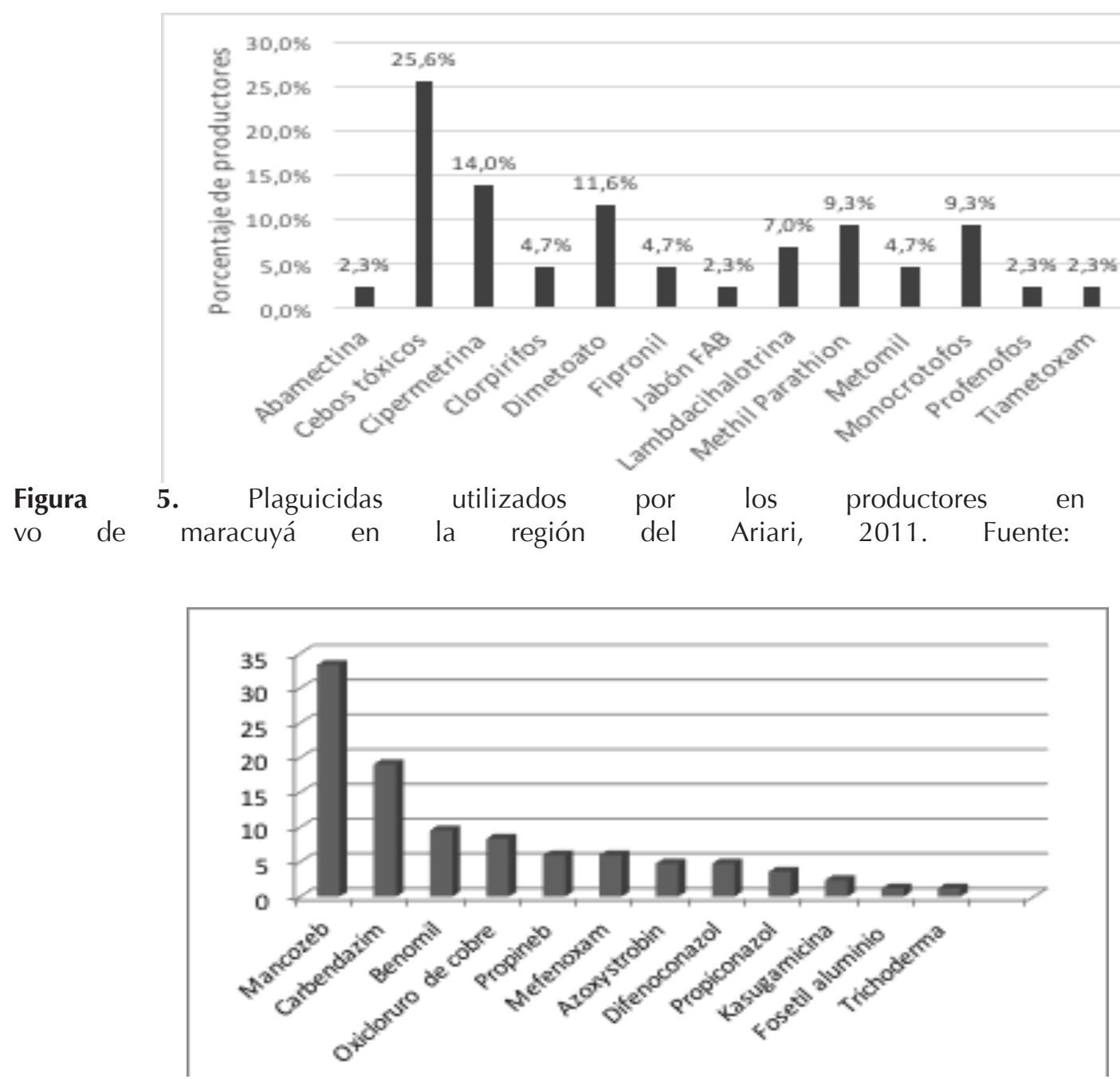

Figura 6. Fungicidas utilizados por los productores en el cultivo de maracuyá para el control de enfermedades en la región del Ariari, 2011. Fuente: Los autores

El cultivo de maracuyá se caracteriza por presentar pocos problemas fitosanitarios limitantes bajo condiciones normales de clima, suelo y humedad, sin embargo, si se altera la situación cambia radicalmente, es por esto que, según el ICA (2011) para llevar a cabo con éxito un programa de manejo de plagas y enfermedades, es necesario ejecutar un control preventivo que se base en el monitoreo constante y en la selección adecuada de prácticas a realizar. De acuerdo a Amaya (2009) los agentes patógenos son los responsables del $25 \%$ de la pudrición en los frutos de maracuyá lo cual genera pérdidas económicas considerables. Por otra parte, afirma que el control de los insectos plaga debe hacerse de manera adecuada con el fin de conservar los insectos polinizadores, por lo tanto, hay que tener especial cuidado en la formulación y dosificación de los insecticidas. De acuerdo a los esquemas de BPA, se hace especial énfasis en la reducción del uso de productos químicos, especialmente aquellos que son tóxicos para las personas y el medio ambiente (Betancurt et al. 2014). Para esto existen alternativas de control, como el uso de microorganismos con capacidad de biocontrol, insectos parásitos y depredadores (ICA, 2011; CIAT, 2012).

Vaslidación y ampliación de la información en los talleres participativos. Las principales dificultades que enfrentan 
los productores para resolver los problemas fitosanitarios son el desconocimiento de los niveles de daño económico de las poblaciones de plagas, y de las herramientas de control biológico y natural, aunado por la escasa información sobre el uso apropiado de agroquímicos. Lo anterior ocasiona que se realicen aplicaciones frecuentes de plaguicidas de forma calendario, se desconocen los períodos de carencia y las plagas que controlan estos plaguicidas, generando así poca eficacia y eficiencia en el manejo de los distintos problemas fitosanitarios. Para el caso particular de los productores de guayaba, estos comentan que casi todos los problemas fitosanitarios los pueden manejar químicamente sin tener pérdidas económicas, pero respecto a los nematodos no saben reconocer los síntomas a tiempo, ni la forma de manejarlo y terminan abandonando el cultivo. Por otro lado, muy pocos productores reciben algún tipo de asistencia técnica o servicios de las instituciones del sector; las recomendaciones sobre el manejo de los cultivos las obtienen de otros productores y de los almacenes de agroquímicos.

Otras limitantes de los cultivos manifestadas por los productores son las siguientes: -Falta de capital: no tienen los recursos económicos suficientes para invertir en mejores alternativas, expandir la producción y desconocen estrategias para la generación de valor agregado de los productos cultivados. -La comercialización: tienen dificultades con los precios bajos en las épocas de cosecha y con ausencia de información para la planificación de los cultivos. -Manejo del suelo: necesitan mejorar la fertilidad y protección de los suelos y el manejo del riego en los meses de verano con el fin de aumentar los rendimientos y ampliar la época de cosecha. -Infraestructura del cultivo de maracuyá: el tutorado para el cultivo tiene un alto costo y dificultades para su consecución, debido a las restricciones de las políticas ambientales para el uso de la madera proveniente del bosque nativo. -Factores climáticos: los cultivos son afectados por los cambios de clima en cuanto a humedad y su efecto sobre las plagas y enfermedades. -Calidad de la semilla: la falta de semillas con características apropiadas y de viveros certificados. -Asistencia técnica y extensión: no poseen acompañamiento de ingenieros agrónomos ni de extensionistas para la implementación de las mejores prácticas de manejo. -Asociatividad: fracasos en las intenciones de asociarse por los trámites burocráticos que estos procesos demandan.

\section{AGRADECIMIENTOS}

El artículo presenta los resultados del proyecto: "Plan de manejo para los cultivos de maracuyá y guayaba" ejecutado por el CIAT y Corpoica; y que fue financiado por el Ministerio de Agricultura y Desarrollo Rural de Colombia. Los autores agradecen a los siguientes investigadores de las instituciones ejecutoras que participaron en el trabajo de campo y en el desarrollo del proyecto: Alonso Gonzalez, Elizabeth Alvarez, German Ceballos y Kris Wyckhuys de CIAT; Laura Arango y Guillermo León de Corpoica (Agrosavia); y al ingeniero agrónomo Lisimaco Vargas contratado por el proyecto.

\section{CONCLUSIONES}

Las condiciones sociodemográficas de los agricultores limitan las capacidades de manejo tecnificado deestos cultivos, ya queen su mayoría son personas con escasa escolaridad que se dedican a la producción para su propio sustento.

La falta de capacitación y conocimiento sobre el manejo adecuado del cultivo es evidente en la mayoría de las unidades productivas, donde el mayor desconocimiento se centra en el uso adecuado de los agroquímicos y en alternativas de control para las plagas y enfermedades. Para el control de plagas y enfermedades 
en ambos cultivos predomina el uso de agroquímicos, los cuales no son aplicados ni almacenados adecuadamente e incluso algunos de ellos tienen componentes de alta toxicidad, lo cual expone la salud de los productores y del medio ambiente en general.

La investigación y la innovación tecnológica puede llevar a mejorar las condiciones de los productores, mediante la estandarización de prácticas adecuadas y de métodos de control diversos y más pertinentes para el control, procurando continuamente la preservación de la salud de las personas y la conservación del medio ambiente.

\section{REFERENCIAS}

AGRONET, Ministerio de Agricultura y Desarrollo Rural. 2017. Área cosechada y producción nacional del cultivo de Guayabo. http:// www.agronet.gov.co/estadistica/ Paginas/Precios.aspx [07-01-2018].

Amaya, R. 2009. "El cultivo del maracuyá" Passiflora edulis form. Flavicarpa. Gerencia Regional Agraria La Libertad, Trujillo-Perú, p30.

Banco Mundial. 2008. Informe sobre el Desarrollo Mundial 2008: Agricultura para el desarrollo. Mayol Ediciones S.A., Bogotá, Colombia, p303.

Betancurt, E., García, E., Giraldo, M., Quejada, O., Rodríguez, H. y Arroyave, I. 2014. Manual técnico del cultivo de maracuyá bajo buenas prácticas agrícolas. Servicio Nacional de Aprendizaje (SENA). Medellín-Colombia.

Brechelt, A. 2004. El manejo ecológico de plagas y enfermedades. Red de Acción en Plaguicidas y sus Alternativas para América Latina (RAP-AL). Fundación Agricultura y Medio Ambiente (FAMA). RD. República Dominicana. p36.
Cadena Productiva Frutícola. 2006. Manual técnico del cultivo de maracuyá (Passiflora edulis L.). Neiva: Secretaría técnica.

CENTA. Centro Nacional De Tecnología Agropecuaria Y Forestal "Enrique Álvarez Córdova". 2010a. Guía técnica del cultivo de la guayaba. Programa MAGCENTA-FRUTALES. El Salvador, p32.

CENTA. Centro Nacional De Tecnología Agropecuaria Y Forestal “Enrique Álvarez Córdova". 2010b. Guía técnica del cultivo de la Maracuyá. Programa MAGCENTA-FRUTALES. El Salvador, p36.

CIAT. Centro Integral de Agricultura Tropical. Productividad agrícola de Colombia: retos y temas pendientes $2012 . \quad$ http://dapa.ciat.cgiar.org/ productividad-agricola-de-colombiaretos-y-temas-pendientes/ [23-08-2016].

CIAT. Centro Integral de Agricultura Tropical. 2012. Cultivo de maracuyá (assiflora edulis f. flavicarpa) establecido con buenas prácticas agrícolas (BPA) en el Centro Internacional de Agricultura Tropical, CIAT. Cali, Colombia, p57.

DANE-CNA. 2016. 3er Censo Nacional Agropecuario: Hay campo para todos. Tomo 2: resultados. GIT Área de Comunicación DANE. Bogotá D. C., Colombia, p1036.

De Rotterdam, C. 2004. Convenio de Rotterdam sobre el procedimiento de consentimiento fundamentado previo aplicable a ciertos plaguicidas y productos químicos peligrosos objeto de comercio internacional. Programa de las Naciones Unidas para el Medio Ambiente y Organización de las Naciones Unidas para la Agricultura y la Alimentación. Rotterdam, Holanda.

El-argawy, E. 2016. Characterization and control of Pestalotiopsis spp. the causal fungus of guava scabby canker in elbeheira governorate, Egypt. International Journal of Phytopathology, 4(3): 121-136. 
Escudero, N., Ferreira, S., Lopez, F., Naranjo, M., Marin, A., Thornton, C., Lopez, L. 2016. Chitosan enhances parasitism of Meloidogyne javanica eggs by the nematopagous fungus Pochonia chlamydosporia. Fungal biology, 120: 572-585.

FAO. Food and Agriculture Organization of the United Nations. 2002. Perspectivas para el medio ambiente Agricultura y medio ambiente. Agricultura mundial: hacia los años 2015 / 2030 Informe resumido, p 75-81.

Gómez, E. y Rebolledo, N. 2006. Modulo del cultivo de Guayaba. Corpoica, p35.

ICA. Instituto Colombiano Agropecuario. 2011. El cultivo de Maracuyá (assiflora edulis) en temporada invernal. Produmedios, Bogotá D. C. Colombia, p35.

ICA. Instituto Colombiano Agropecuario. 2012. Manejo fitosanitario del cultivo de guayaba (Psidium guajava, L.). Medidas para la temporada invernal. Produmedios, Bogotá D. C. Colombia, 28.

ICA. Instituto Colombiano Agropecuario. 2016. Estadísticas de comercialización de plaguicidas químicos de uso agrícola 2015. Boletín técnico. Produmedios, Bogotá D. C. Colombia, p127.

ICA Instituto Colombiano Agropecuario y CORPOICA Corporación Colombiana de Investigación Agropecuaria. 2006. Manejo Fitosanitario del cultivo de la Guayaba (Psidium guajava L.) en Santander. Imprenta Nacional de Colombia. Colombia, p 40.

Izquierdo, J. y Rodríguez Fazzone, M. 2016. Buenas Prácticas Agrícolas: en busca de sostenibilidad, competitividad y seguridad alimentaria. Grupo de Agricultura Oficina Regional de la FAO para América Latina y el Caribe. Santiago, Chile. p66.
Kaur, T., Jasrotia, S., Ohri, P. y Manhas, R. K. 2016. Evaluation of in vitro and in vivo nematicidal potential of a multifunctional streptomycete, Streptomyces hydrogenans strain DH16 against Meloidogyne incognita. Microbiological research, 192: 247-252.

Orduz, J. y Rangel, J. 2002. Frutales tropicales potenciales para el piedemonte Ilanero. Manual de asistencia técnica No. 8. Corporación Colombiana de Investigación Agropecuaria (CORPOICA). Villavicencio, Colombia, p133

Pérez, R., Mitchell, S. y Vargas, R. 2008. Psidium guajava: A review of its traditional uses, phytochemistry and pharmacology. Journal of Ethnopharmacology, 117: 1-27.

Perfetti, J., Hernández, A., Leibovich, J., y Balcázar, A. 2013. Políticas para el desarrollo de la agricultura en Colombia. SAC y Fedesarrollo. Bogotá, D.C., p248.

Pingali, P. 2007. Agricultural Growth and Economic Development: a view through the globalization lens. Agricultural Economists 37:1-12.

Prakash, O. y Pandey, 2007. Current scenario of guava diseases in India and their integrated management. Acta Horticulturae 735: 495-505.

Salazar, D., Melgarejo, P., Martinez, R., Martinez, J., Hernandez, F. y Burguesa, M. 2006. Phenological states of the guava tree (Psidium guajava L.). Scientia Horticulturae, 108: 157-161.

Zhang, S., Gan, Y. y Xu, B. 2015. Biocontrol potential of native species of Trichoderma longibrachiatum against Meloidogyne incognita. Applied Soil Ecology, 94:21-29.

Zibadi, S. and Watson, R. 2004. Passion fruit (assiflora edulis). Evidence-Based Integrative Medicine, 1(3), 183-187. Springer. 